OPEN ACCESS

Edited by:

Ricardo De La Vega,

Autonomous University of

Madrid, Spain

Reviewed by:

Jon Irazusta,

University of the Basque

Country, Spain

Raul Antunes,

Polytechnic Institute of Leiria, Portugal

*Correspondence:

Elaine A. Hargreaves elaine.hargreaves@otago.ac.nz

Specialty section:

This article was submitted to

Health Psychology,

a section of the journal

Frontiers in Psychology

Received: 17 December 2020

Accepted: 29 January 2021

Published: 25 February 2021

Citation:

Hargreaves $E A$, Lee $C$, Jenkins $M$, Calverley JR, Hodge $K$ and Houge

Mackenzie S (2021) Changes in

Physical Activity Pre-, During and Post-lockdown COVID-19 Restrictions in New Zealand and the Explanatory Role of Daily Hassles.

Front. Psychol. 12:642954

doi: 10.3389/fpsyg.2021.642954

\section{Changes in Physical Activity Pre-, During and Post-lockdown COVID-19 Restrictions in New Zealand and the Explanatory Role of Daily Hassles}

\author{
Elaine A. Hargreaves ${ }^{1 *}$, Craig Lee ${ }^{2}$, Matthew Jenkins ${ }^{3}$, Jessica R. Calverley ${ }^{1}$, Ken Hodge ${ }^{1}$ \\ and Susan Houge Mackenzie ${ }^{2}$
}

${ }^{1}$ School of Physical Education, Sport \& Exercise Sciences, University of Otago, Dunedin, New Zealand, ${ }^{2}$ Department of Tourism, University of Otago, Dunedin, New Zealand, ${ }^{3}$ Department of Psychological Medicine, University of Otago, Wellington, New Zealand

Covid-19 lockdown restrictions constitute a population-wide "life-change event" disrupting normal daily routines. It was proposed that as a result of these lockdown restrictions, physical activity levels would likely decline. However, it could also be argued that lifestyle disruption may result in the formation of increased physical activity habits. Using a longitudinal design, the purpose of this study was to investigate changes in physical activity of different intensities, across individuals who differed in activity levels prior to lockdown restrictions being imposed, and across three time periods: pre-, during- and post-lockdown. This study also examined the extent to which the experience of daily hassles explained any changes in physical activity. A convenience sample $(N=759)$ recruited through social media, provided data from an online survey administered during weeks 2-3 of a 5-week lockdown and 231 participants provided complete data again 6 weeks post-lockdown (72\% female, $M$ age $=43$ years). Participants completed the International Physical Activity Questionnaire-Short Form and the Daily Hassles Scale. Results showed that vigorous and moderate intensity PA were significantly lower during- and post-lockdown compared to pre-lockdown in those individuals who had been highly active pre-lockdown. In contrast, for moderately active individuals pre-lockdown, vigorous and moderate intensity PA was significantly higher during-lockdown compared to pre-lockdown, and these increased levels of vigorous PA were maintained post-lockdown. Participants experienced daily hassles due to inner concerns, time pressures, family, and financial concerns to the same extent during- and post-lockdown. Those daily hassles had a small negative (Standardized $\beta=-0.11$; $p<0.05)$ predictive effect on post-lockdown PA. It appears that to understand the effect of COVID-19 restrictions on PA, the activity status of individuals pre-lockdown needs to be taken into account. The daily hassles appeared to play a role in post-lockdown PA behavior, but future research should investigate why these results occurred.

Keywords: behavior change, exercise, psychology, stress, physical activity intensity, CoVID-19 


\section{INTRODUCTION}

In March 2020, the New Zealand (NZ) Government instigated Level 4 lockdown restrictions in response to the World Health Organization (WHO) declared COVID-19 pandemic (World Health Organisation, 2020). These Level 4 lockdown restrictions (henceforth termed "lockdown") urged all New Zealanders (except those classed as essential workers) to stay at home unless undertaking a limited range of "essential" activities (e.g., shopping for groceries, medical reasons) (New Zealand Government, 2020a). Importantly, the NZ government allowed individuals to be physically active in their local neighborhood, imparting to the NZ public the importance of being physically active for health and well-being during this period (New Zealand Government, 2020b). The physiological and psychological benefits (e.g., reductions in depression and anxiety levels) of being physically active are well-recognized (World Health Organization, 2010), and more specific benefits related to the effects of COVID-19 have been proposed (e.g., Matias et al., 2020; Simpson and Katsanis, 2020; Woods et al., 2020). In particular, the COVID-19 pandemic and its associated restrictions have resulted in substantial psychosocial effects, for example increased prevalence of anxiety and stress (Salari et al., 2020), which physical activity (PA) is known to ameliorate (Stubbs et al., 2017). Consequently, researchers have opinionated on the importance of being physically active during the pandemic (Chen et al., 2020; Hudson and Sprow, 2020; Lippi et al., 2020; Ricci et al., 2020; Sallis and Pratt, 2020).

The NZ lockdown constituted a significant lifestyle change for individuals. Many people were either not working or were working from home, plus schools, indoor exercise and recreation facilities were closed, club/community sport was canceled, and outdoor recreation was limited to local neighborhoods. These unprecedented conditions constituted a population-wide "life-change event," defined by the US National Library of Medicine as "those occurrences, including social, psychological and environmental, which require an adjustment or effect a change in an individual's pattern of living" (Engberg et al., 2012, p. 433). Life-change events disrupt a person's daily routine and are a known determinant of physical activity (PA) behavior change (Engberg et al., 2012). This particular life-change event may have facilitated old habits being broken (e.g., patterns of inactivity) and the formation of new habits (e.g., adoption of PA), in part because time-related barriers for PA may be removed. Alternatively, the lockdown restrictions may have decreased normal physical activity levels (e.g., due to exercise facilities being closed, or recreational areas being unavailable) or made people less likely to be physical active (e.g., due to increased childcare demands, or COVID-related anxiety). Consequently, investigating how PA changed in the NZ context as a result of the lockdown provides valuable insights on the ramifications for health-related behavior.

At the outset of the COVID-19 pandemic, researchers suggested PA levels would decline (e.g., Papaioannou et al., 2020). In 2020, studies from different countries (e.g., Belgium, Canada, Greece, USA, Australia) investigated changes in PA behavior as a result of their varied COVID-19 restrictions.
Most of these studies used cross-sectional designs with online survey methods, collecting data at one point during lockdown along with retrospective assessment of PA prior to COVID-19 restrictions being implemented. PA behavior was assessed in different ways; for example, with validated self-report measures, non-validated measures or a single item question asking whether PA changed. Summarizing this research, PA was shown to be lower during COVID-19 restrictions compared to before restrictions were put in place (López-Bueno et al., 2020; Mutz and Gerke, 2020; Schnitzer et al., 2020; Stanton et al., 2020). More nuanced analysis has shown that between 24 and $49 \%$ of samples had decreased PA levels during restrictions, $21-32 \%$ had increased PA levels, and $30.5-44 \%$ had no change in PA (Brand et al., 2020; Knell et al., 2020; Mutz and Gerke, 2020; Stanton et al., 2020).

Other research has shown that changes in PA differed as a function of individuals' pre-lockdown PA levels. Meyer et al. (2020) found $32 \%$ of their US sample who were active preCOVID restrictions decreased their PA levels during restrictions, while PA levels remained unchanged for those who were inactive prior to restrictions. Barkley et al. (2020) used tertile splits based on PA scores pre-COVID restrictions from the Godin LeisureTime Exercise Questionnaire (Godin and Shephard, 1985) to create, low, moderate and high active groups. They found that US university staff and students classified as high active (average of $75.8 \mathrm{METs} /$ week) reduced their PA during the COVID-19 restrictions by $22 \%$; those classed as moderately active (average of $32.5 \mathrm{METs} /$ week) increased their PA by $14 \%$; and those classed as least active (12.9 METs/week) increased their PA by $83 \%$. Also using the Godin questionnaire, Lesser and Nienhuis (2020) found, of the $63 \%$ of Canadians classed as inactive pre-COVID restrictions (participating in $<150 \mathrm{~min} /$ week of moderate to vigorous $\mathrm{PA}$ ), $40.5 \%$ became less active, and $33 \%$ became more active during the restrictions. Of the $37 \%$ classed as active pre-COVID restrictions (participating in $\geq 150 \mathrm{~min} /$ week of moderate to vigorous PA), $22.4 \%$ became less active and $40.3 \%$ became more active. In Belgium, Constandt et al. (2020) reported that, amongst people classed as "high active" (exercising regularly/at least once a week) prior to COVID-19 restrictions, $36 \%$ exercised more, $23 \%$ exercised less, and $41 \%$ exercised the same during restrictions. Amongst "low active" individuals (exercised non-regularly/less than once a week), 58\% exercised more, $7 \%$ exercised less, $5 \%$ exercised the same, and 30\% did not exercise at all.

Given these mixed findings, further investigation of PA behavior in relation to COVID-19 restrictions is warranted, particularly with the use of a validated self-report measure of PA that has been missing in some studies (e.g., Constandt et al., 2020; Meyer et al., 2020; Mutz and Gerke, 2020) and a classification of activity level that corresponds with the WHO PA guidelines. Additionally, research has yet to investigate potential changes in physical activity intensity during COVID19 restrictions (e.g., vigorous, moderate, walking) and how PA may have changed once lockdown restrictions were eased. NZ provides a unique context in which to study PA after COVID-19 restrictions were lifted because of its success in largely eliminating community transmission which allowed 
people to return to pre-COVID mobility levels (Wilson et al., 2020).

Research is yet to provide psychological-based explanations for why PA changes have occurred during COVID-19 restrictions (Papaioannou et al., 2020; Sallis et al., 2020). Systematic reviews have shown that, in general, life changes/events have a negative effect on PA participation (Allender et al., 2008; Engberg et al., 2012; Stults-Kolehmainen and Sinha, 2014). However, different life events can have differential effects on PA, with some prompting PA increases (e.g., change in employment status) and others prompting PA decreases (e.g., transition to University, having a child) (see Engberg et al., 2012 for review). Researchers have suggested that, because not everyone responds to life events in the same way, it may not be the particular life event that influences behavior change per se, but rather the daily hassles (i.e., stressors) that the event creates in a person's life (Kanner et al., 1981; O'Connor et al., 2009; Uijtdewilligen et al., 2014). For example, DeLongis et al. (1982) showed that a larger percentage of variance in health status was explained by the daily hassles in people's lives resulting from a major life event, rather than stress due to the event itself. Therefore, it may be that the differential effects of COVID-19 restrictions on PA hinges upon the experienced daily hassles created by the event. Indeed, Cheval et al. (2020) suggested a measure of stress should be included in research to examine how it moderates PA change.

Daily hassles have been operationalized as a measure of the everyday stressors (i.e., problems or difficulties that are part of everyday life) that a particular situation causes (O'Connor et al., 2009). The extent of daily hassles has been associated with poorer eating behaviors (O'Connor et al., 2008), lower physical activity (Twisk et al., 1999; Nguyen-Michel et al., 2006) and is predictive of actual stress experienced (Feizi et al., 2012). Nguyen-Michel et al. (2006) found support for their hypothesis that PA would be associated with lower perceived daily hassles, but identified that it was equally plausible that the experience of daily hassles would discourage physical activity participation. In their review of the PA and stress research, StultsKolehmainen and Sinha (2014) also found evidence for these bidirectional relationships. Physical activity status may also protect people from experiencing stress as a result of the daily hassles experienced (Feizi et al., 2012). Thus, how active individuals are may influence their response to the daily hassles caused by the COVID-19 restrictions and provide an explanation for PA changes.

The purposes of this study were 2 -fold. Firstly, we examined changes in PA from pre-lockdown to PA during-lockdown, and post-lockdown. Specifically, we aimed to examine changes in total PA and PA of different intensities, and compared the PA of individuals who met the WHO PA guidelines pre-lockdown (deemed moderately active), exceeded the PA guidelines (deemed highly active) and who did not meet the guidelines (deemed inactive). We made tentative hypotheses that overall physical activity would decrease during lockdown compared to prelockdown, but recover to pre-lockdown levels post-lockdown. We expected highly and moderately active individuals to remain active during- and post-lockdown, and for inactive individuals to increase their PA during- and post-lockdown. The analyses on the changes to PA of different intensities were exploratory. Secondly, we investigated the experience of daily hassles during- and postlockdown and the extent to which those daily hassles predicted PA during- and post-lockdown. We hypothesized that the experience of daily hassles would be higher during-compared to post-lockdown. We expected that daily hassles would negatively predict PA behavior, but this result would be moderated by the individuals' pre-lockdown PA status.

\section{MATERIALS AND METHODS \\ Participants and Procedures}

A convenience sample of the NZ population was recruited using social media recruitment methods (King et al., 2014) and a virtual snowball recruitment technique (Baltar and Brunet, 2012). Study information with a link to an online survey (hosted on the online survey platform Qualtrics) was shared via Facebook and circulated via email to contacts at NZ universities and other organizations (e.g., regional and national sports organizations, city councils) for dissemination through their networks. The study advertising specifically stated that we wanted to hear from people who were not overly active as well as those who were active in an effort to reduce response bias. Participants were eligible if they were aged 18 years or older and living in NZ for the duration of the lockdown restrictions. Those with any contraindications (e.g., illness, injury) that prevented being physically active during the lockdown restrictions were excluded. These criteria were assessed via screening questions at the beginning of the online survey.

Participants completed the survey at two time points. The first was completed between the second and third weeks of NZ's 5week lockdown period (April 8 to April 15, 2020; the survey was open for 8 days). We deemed this data point "duringlockdown". Secondly, those participants who completed the first survey and agreed to be contacted again were emailed the link to the follow-up survey. The follow-up survey was sent on June 9th 2020 and was open for completion for 9 days. Participants were sent a reminder 6 days after the first email was sent. This period was 6 weeks after lockdown had ended and was the final week of four where NZ was subject to Level 2 restrictions. Under these restrictions, people could leave home and travel freely, and were asked to adhere to public health measures (e.g., physical distancing, mask wearing on public transport). All gyms, health clubs and swimming pools were open with public health measures in place. Community sport was also allowed but restricted to a maximum of 100 people in a confined space. We deemed this point "post-lockdown". Ethical approval was obtained from the university ethics committee (reference: D20/214). All participants provided online informed consent before completing the survey. This study comprises part of a larger project investigating determinants and outcomes of PA as a result of the COVID-19 lockdown restrictions. The full project survey took an average of $12 \mathrm{~min}$ to complete.

The during-lockdown survey elicited 759 responses. Of those 759,464 agreed to be contacted to complete the post-lockdown survey and 352 surveys were returned. After data cleaning (see Figure 1), the final sample for analysis consisted of 231 


\section{Time 1: During-lockdown} survey distributed

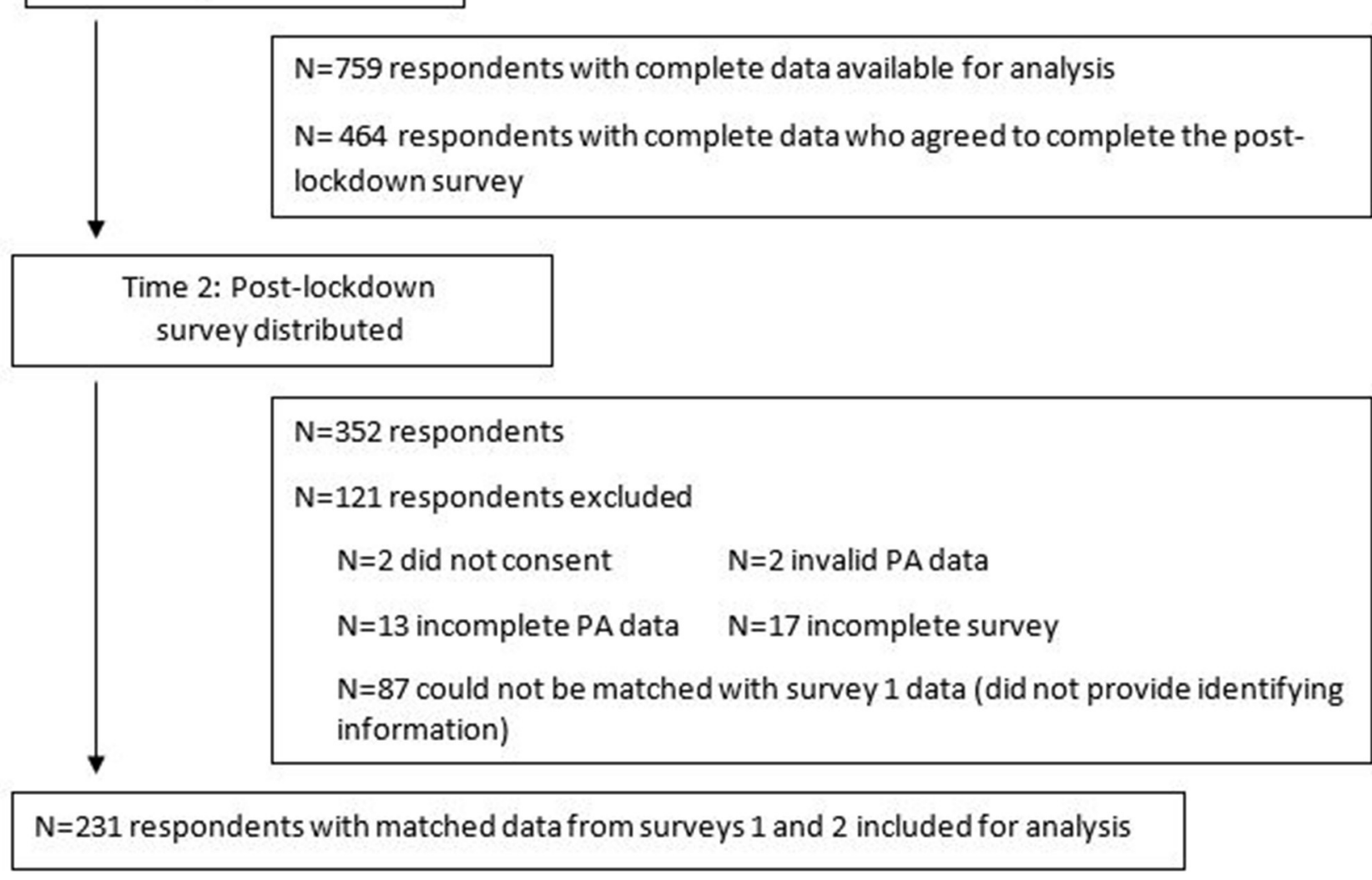

FIGURE 1 | Survey responses during-lockdown (Time 1) and post-lockdown (Time 2).

responses. Detailed participant characteristics of those who responded during-lockdown and the 231 with data from both surveys is shown in Table 1. Sample participants ranged in age from 18 to 81 years (mean $=42.91, \mathrm{SD}=13.84$ ), were predominantly female (72.3\%), NZ European (82.7\%), and with at least a university degree $(75.8 \%)$. Most were not essential workers $(85.3 \%)$.

\section{Measures}

\section{Physical Activity}

The International Physical Activity Questionnaire-Short Form (IPAQ-SF; Craig et al., 2003) is a 7-item measure of selfreported PA, measuring the amount of moderate- and vigorousintensity PA and walking undertaken by participants over the previous 7 days. Example items include: "During the last 7 days, on how many days did you do vigorous activities like heavy lifting, exercise classes, or fast cycling for at least $10 \mathrm{~min}$ at a time?" followed by the question: "How much time did you usually spend doing vigorous physical activities on one of those days?". The IPAQ-SF has demonstrated good validity and consistency (Lee et al., 2011). For the during-lockdown survey participants completed the IPAQ-SF twice. Firstly, they were asked to report their PA from the previous week (duringlockdown). Secondly, they were asked to report their PA from a typical week prior to lockdown. To lessen the risk of recall bias we ensured that the IPAQ-SF was used in the form in which it had been validated and that it incorporated examples of moderate, and vigorous intensity activities (Matthews et al., 2012). In the post-lockdown survey, they were only asked to report their PA from the previous week (during level 2 postlockdown restrictions).

Data screening, cleaning, and coding were undertaken according to detailed guidelines (IPAQ group, 2014). This included the truncation of data points indicating more than $960 \mathrm{~min} /$ week $(16 \mathrm{~h})$ as these are suggested to be outliers. Participants were classified as moderately, or highly active, or inactive according to proposed guidelines (IPAQ group, 2014). To be classified as moderately active, participants met one of the following criteria: (a) $\geq 3$ days of vigorous intensity activity of $\geq 20$ min per day; (b) $\geq 5$ days of moderate intensity activity and/or walking of at least $30 \mathrm{~min}$ per day; or (c) $\geq 5$ days of any combination of walking, moderate intensity or vigorous intensity activities achieving $\geq 600 \mathrm{MET} \mathrm{min/week.} \mathrm{The} \mathrm{highly}$ active participants achieved $\geq 1,500$ or $\geq 3,000 \mathrm{MET}$ min/week, 
TABLE 1 | Participant characteristics.

\begin{tabular}{lcc}
\hline & $\begin{array}{c}\text { Respondents } \\
\text { during-lockdown }\end{array}$ & $\begin{array}{c}\text { Respondents with } \\
\text { data from during- \& } \\
\text { post-lockdown }\end{array}$ \\
\hline Mean age (SD) years & $53(14)$ & $43(14)$ \\
Gender [n (\%)] & $195(26.2)$ & $167(72.3)$ \\
Female & $3(0.4)$ & $63(27.3)$ \\
Male & $3(0.4)$ & $1(0.4)$ \\
Gender diverse & $574(82.9)$ & 0.0 \\
Prefer not to say & $6(0.9)$ & $191(82.7)$ \\
Ethnicity [n (\%)] & $1(0.1)$ & $2(0.9)$ \\
NZ European & $2(0.3)$ & 0.0 \\
Māori & $4(0.6)$ & 0.0 \\
Samoan & $2(0.3)$ & 0.0 \\
Cook Island Māori & $91(13.2)$ & $1(0.4)$ \\
Chinese & $12(1.7)$ & $36(15.6)$ \\
Indian & $102(13.8)$ & $1(0.4)$ \\
Other & & $34.7)$ \\
Prefer not to say & & \\
Essential worker (N Yes, \%) & & \\
\hline
\end{tabular}

depending on intensity of their PA and the inactive participants did not meet the criteria for moderate or high PA. PA was calculated as weekly total PA MET mins; weekly moderate intensity MET mins, weekly vigorous intensity MET mins and weekly MET mins of walking according to proposed guidelines (IPAQ group, 2014).

\section{Daily Hassles}

The Hassles Scale (Kanner et al., 1981) is a 117-item measure of the daily hassles participants experienced in the last 7 days. Participants were instructed to rate the degree to which each hassle item had affected their life in the past seven days (i.e., during-lockdown for the first survey administration, and postlockdown for the second survey administration). Participants responded by selecting, "This is not a hassle for me" (0), "A somewhat severe hassle" (1), "A moderately severe hassle" (2) or "An extremely severe hassle" (3) for each item. Following Holm and Holroyd (1992), we grouped the daily hassle items according to seven categories: inner concerns (example items: troubling thoughts about one's future, trouble relaxing, concerns about inner conflicts), financial concerns (example items: financial security, concerns about job security, concerns about owing money), time pressures (example items: too many things to do, too many responsibilities, too many interruptions), work hassles (example items: job dissatisfaction, worries about decisions to change jobs), environmental hassles (example items: concerns about news events, crime), family hassles (example items: friends or relatives too far away, problems with one's children, health of a family member) and health hassles (example items: concerns about health in general, use of alcohol, concerns about weight). A severity score for each category was created by summing the item scores.

\section{Data Analysis}

To investigate changes in PA intensity from pre- to duringand post-lockdown, in the different activity groups, a factorial repeated measures (PA intensity $\mathrm{x}$ time $\mathrm{x}$ activity group) ANOVA was conducted. To compare changes in the daily hassles categories between during-lockdown and post-lockdown, a factorial repeated measures ANOVA (daily hassles severity $\mathrm{x}$ time) was conducted. The Bonferroni procedure was used for post-hoc tests in both ANOVAs. Finally, to examine the extent to which severity of daily hassles predicted total PA during and after the lockdown, and whether any effects were moderated by activity level of participants pre-lockdown (activity group), we conducted two hierarchical multiple regressions-one using during-lockdown data and the second using post-lockdown data. In both analyses, the control variables of pre-lockdown total PA MET mins/week, age, gender, education, ethnicity, whether the respondent was an essential worker, and the number of dependent children were entered into the model first (Model 1). The severity of daily hassles during- or post-lockdown was then entered into the model as the direct predictor of total weekly PA (Model 2). Finally, the interaction between PA group and the extent of daily hassles was entered (Model 3).

\section{RESULTS}

Of the 231 people who provided valid PA data pre-, during-, and after-lockdown, 111 were categorized as being highly active prelockdown and 120 as moderately active pre-lockdown. Although 32 inactive participants completed the during-lockdown survey, they did not complete the post-lockdown survey.

\section{Change in PA}

\section{Highly Active Group (Pre-lockdown)}

There was a significant main effect of the lockdown restrictions on total PA $\left[F_{(2,220)}=36.88, p<0.001\right.$, partial eta $^{2}=$ 0.40]. The follow-up Bonferroni post-hoc tests revealed that PA MET mins/week before lockdown were significantly higher than during-lockdown and post-lockdown. There was no significant difference between total PA during-lockdown and post-lockdown (see Table 2 for mean values).

There was also a significant main effect of PA intensity $\left[F_{(1.51,166.45)}=216.61, \varepsilon=0.76, p<0.001\right]$, and an interaction for PA intensity and time $\left[F_{(3.35,368.57)}=13.59\right.$, partial eta ${ }^{2}$ $=0.11$ ] on total PA MET mins/week. The interaction results showed that lockdown restrictions significantly affected vigorous intensity $\left[F_{(2,220)}=30.07, p<0.001\right.$, partial eta $\left.{ }^{2}=0.22\right]$ and moderate PA $\left[F_{(2,220)}=9.87, p<0.001\right.$, partial eta $\left.{ }^{2}=0.08\right]$, but not walking $\left[F_{(2,220)}=1.493, p=0.23\right]$. The Bonferroni posthoc tests showed that vigorous PA pre-lockdown was significantly higher than during-lockdown and post-lockdown. There was no significant difference between during- and post-lockdown. Moderate PA pre-lockdown was significantly higher than duringlockdown and post-lockdown $(M=432.51, \mathrm{SE}=26.57)$. There was no significant difference between during- and post-lockdown (see Table 2 for mean values).

Thus, for individuals classed as highly active pre-lockdown, their vigorous and moderate intensity PA levels dropped during 
TABLE 2 | Mean (SE) physical activity pre-lockdown, during-lockdown, and post-lockdown.

\begin{tabular}{|c|c|c|c|c|c|c|}
\hline & \multicolumn{2}{|c|}{ Pre-lockdown } & \multicolumn{2}{|c|}{ During-lockdown } & \multicolumn{2}{|c|}{ Post-lockdown } \\
\hline & $\begin{array}{l}\text { Highly } \\
\text { active }\end{array}$ & $\begin{array}{l}\text { Moderately } \\
\text { active }\end{array}$ & $\begin{array}{l}\text { Highly } \\
\text { active }\end{array}$ & $\begin{array}{l}\text { Moderately } \\
\text { active }\end{array}$ & $\begin{array}{l}\text { Highly } \\
\text { active }\end{array}$ & $\begin{array}{c}\text { Moderately } \\
\text { active }\end{array}$ \\
\hline $\begin{array}{l}\text { Total PA } \\
\text { (MET mins/week) }\end{array}$ & $2,290(40)$ & $868(38)$ & $1,794(64)$ & $1,218(66)$ & $1,808(66)$ & $1,018(62)$ \\
\hline $\begin{array}{l}\text { Vigorous intensity } \\
\text { (MET mins/week) }\end{array}$ & $1,285(28)$ & $233(29)$ & $951(52)$ & $496(50)$ & $936(52)$ & 395 (46) \\
\hline $\begin{array}{l}\text { Moderate intensity } \\
\text { (MET mins/week) }\end{array}$ & $543(24)$ & $253(21)$ & $419(27)$ & 289 (26) & $433(27)$ & $244(24)$ \\
\hline Walking (MET mins/week) & $462(18)$ & $382(20)$ & $424(20)$ & 434 (19) & $439(20)$ & $379(21)$ \\
\hline
\end{tabular}

lockdown, and remained at this level post-lockdown. While, walking met mins/week stayed the same throughout the three time periods (see Figure 2).

\section{Moderately Active Group (Pre-lockdown)}

There was a significant time main effect for total PA MET mins/week $\left[F_{(2,238)}=14.92, p<0.001\right.$, partial eta $\left.{ }^{2}=0.11\right]$. The Bonferroni post-hoc tests revealed that total PA duringlockdown was significantly higher than pre-lockdown and postlockdown. Also, post-lockdown PA was significantly higher than pre-lockdown. There was also a significant main effect for PA intensity $\left[F_{(1.57,187.07)}=11.74, \varepsilon=0.79, p<0.001\right]$, showing vigorous $\mathrm{PA}$ and walking were significantly higher than moderate PA $(M=261.92, \mathrm{SE}=17.59)$. There was no significant difference between vigorous $\mathrm{PA}$ and walking.

There was a significant interaction for PA intensity and time $\left[F_{(3.2,380.96)}=7.34, p<0.001\right.$, partial eta $\left.{ }^{2}=0.06\right]$. The results showed that vigorous PA $\left[F_{(2,238)}=14.18, p<0.001\right.$, partial eta ${ }^{2}$ $=0.11]$, moderate PA $\left[F_{(1.41,168.21)}=20.82, p<0.001\right.$, partial eta $\left.^{2}=0.15\right]$ and walking $\left[F_{(2,238)}=3.54, p \leq 0.03\right.$, partial eta ${ }^{2}=$ 0.03 ] were affected by the lockdown restrictions. The Bonferroni post-hoc tests showed that vigorous PA during-lockdown and post-lockdown was significantly higher than pre-lockdown. There was no significant difference between during- and postlockdown. Moderate PA during-lockdown was significantly higher than pre-lockdown and post-lockdown. There was no significant difference between pre- and post-lockdown. Duringlockdown walking was significantly higher than post-lockdown walking. There was no significant difference in walking between pre-lockdown and during- or post-lockdown.

Thus, for individuals classed as moderately active pre-lockdown, their participation in vigorous and moderate intensity PA increased during lockdown compared to before. Post-lockdown, they maintained their vigorous intensity PA, but moderate intensity PA returned to pre-lockdown levels. While walking did not change from pre- to duringlockdown, it reduced from during- to post-lockdown (see Figure 2).

\section{Experience of Daily Hassles}

There were no significant differences in the severity of each daily hassle category between during- and post-lockdown (See Figure 3 and Table 3). Despite this, the scores show that the predominant daily hassles experienced during- and post-lockdown were inner concerns, time pressures, family hassles, and financial concerns.

\section{Relationship Between Daily Hassles and Physical Activity During-Lockdown}

Model 1, with only the control variables as predictors, explained $22 \%$ of the variance in PA behavior during-lockdown $\left[F_{(7,223)}=\right.$ $8.97, p<0.0001]$. Model 2 , with the extent of daily hassles duringlockdown included as a predictor, explained $22.3 \%$ of the variance in PA behavior during-lockdown $\left[F_{(8,222)}=7.98, p<0.001\right]$. This represented a non-significant improvement of $0.3 \%$ over Model $1\left[R^{2} \Delta: F_{(1,222)}=1.06, p<0.30\right]$. Model 3, with the interaction term between the extent of daily hassles during lockdown and PA group included as a predictor, did not make any improvements over Model 3 (see Table 4).

\section{Relationship Between Daily Hassles and Physical Activity Post-lockdown}

Model 1, with the control variables as predictors, explained $48 \%$ of the variance in PA behavior post-lockdown $\left[F_{(8,222)}=25.65\right.$, $p<0.001]$. Model 2, with the severity of daily hassles postlockdown as a predictor, explained $49.1 \%$ of the variance in PA behavior $\left[F_{(9,221)}=23.72, p<0.001\right]$. This represented a significant improvement of $1.1 \%$ over Model $1\left[R^{2} \Delta: F_{(1,221)}\right.$ $=4.77, p<0.05]$. Model 3, which included the interaction term between the severity of daily hassles and PA group as predictors did not make any improvements over Model 3 (see Table 5).

These results indicate that post-lockdown there was a small negative association between severity of daily hassles and PA behavior, but there was no association between daily hassles and PA during-lockdown.

\section{DISCUSSION}

The first purpose of this study was to examine changes in total PA and PA intensity across pre-lockdown, duringlockdown, and post-lockdown COVID-19 restrictions. We aimed to examine changes in those participants classed as low, highly and moderately active pre-lockdown. The second purpose was to investigate the extent to which the experience of daily hassles influenced any change in PA behavior. Results showed that, for 
A

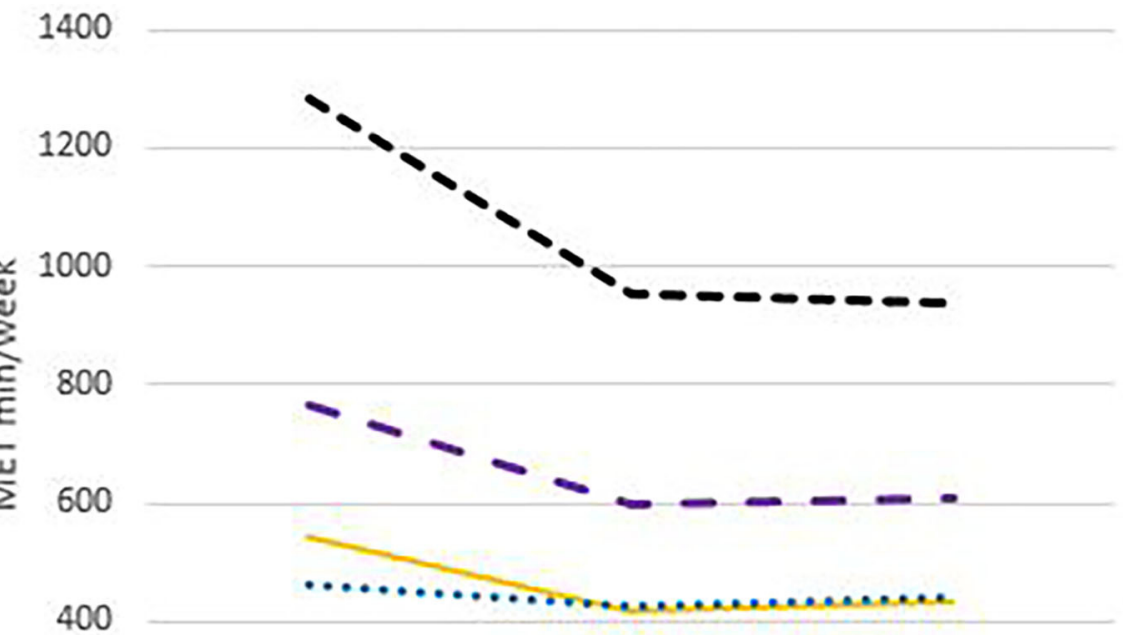

200

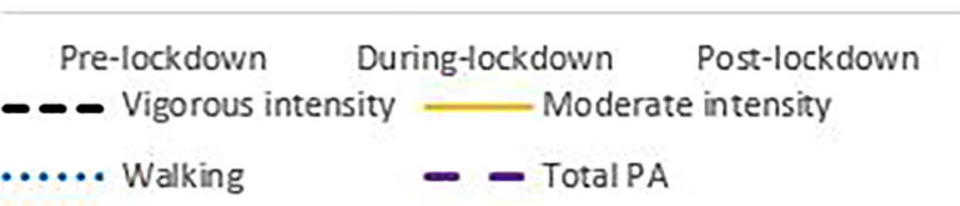

B

550

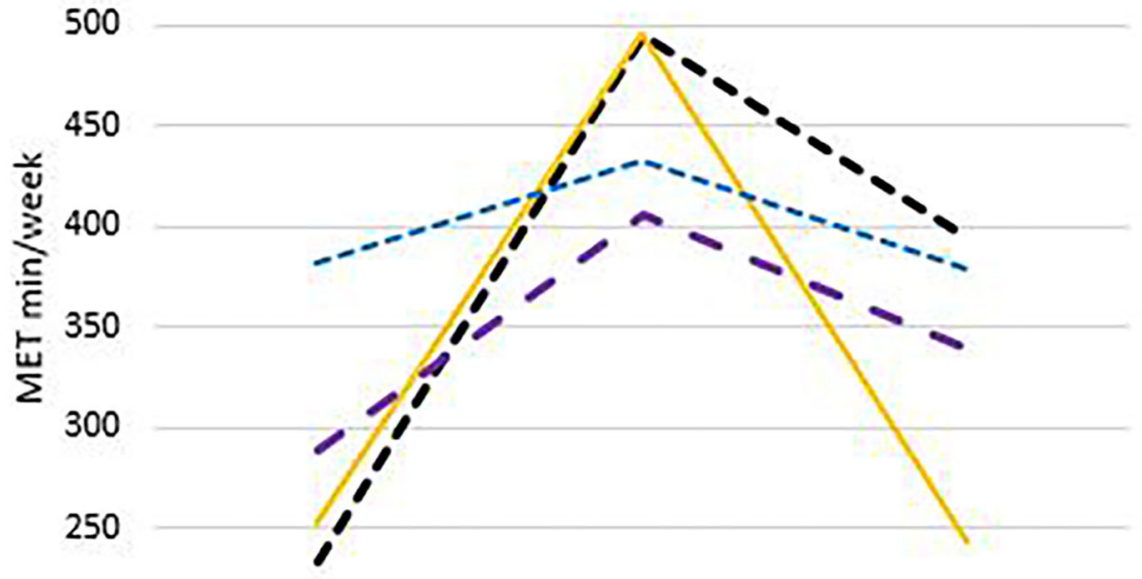

200

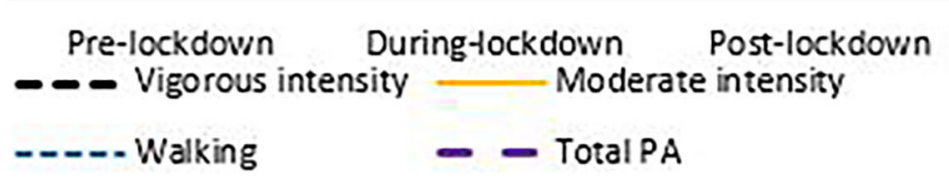

FIGURE 2 | Total PA and vigorous, moderate and walking MET mins/week pre-, during and post-lockdown in highly active (A) and moderately active (B) participants pre-lockdown. 


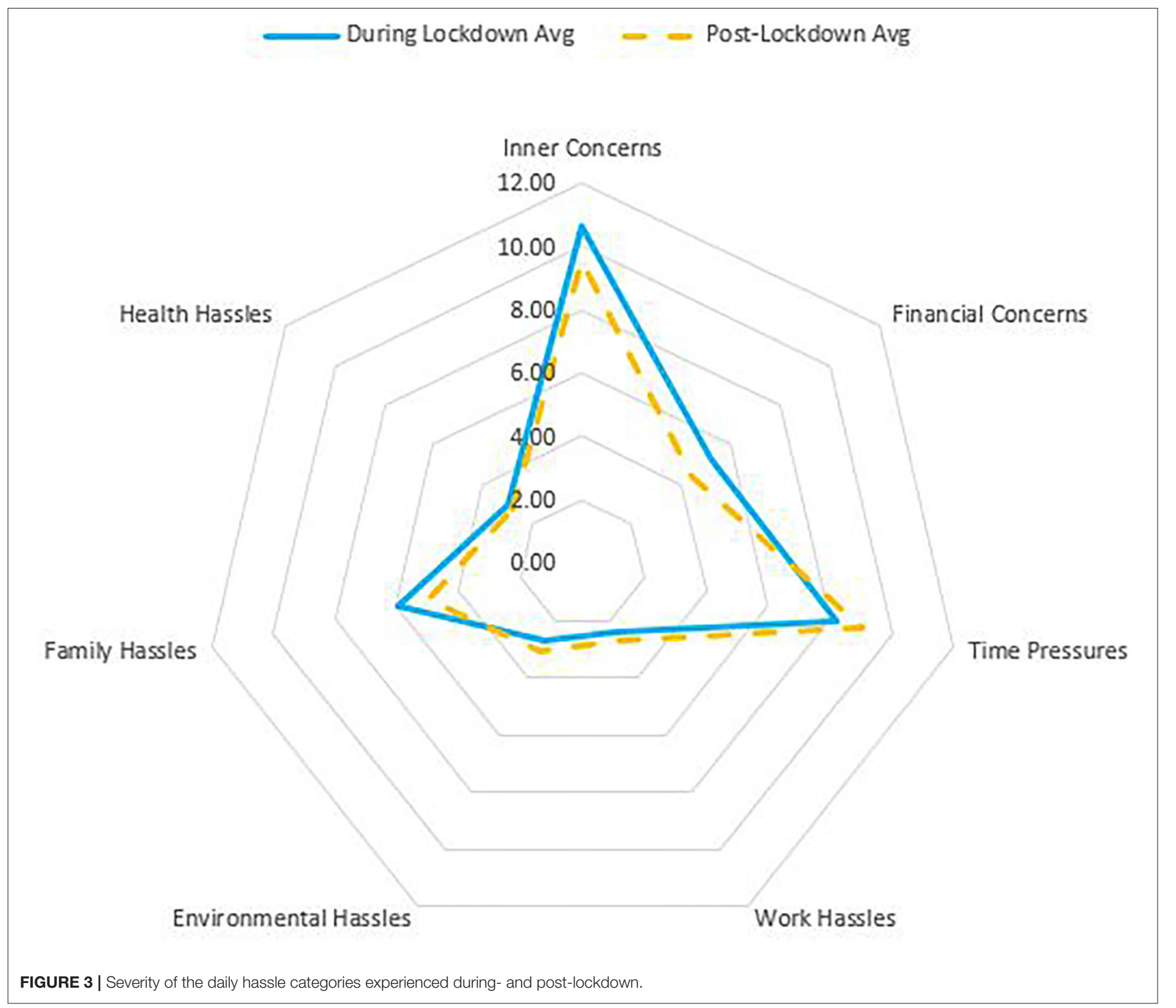

TABLE 3 | Mean (SD) severity of daily hassles experienced and percentage of sample experiencing specific hassles during and post-lockdown.

\begin{tabular}{|c|c|c|c|c|}
\hline \multirow[b]{2}{*}{ Daily Hassle category } & \multicolumn{2}{|c|}{ During lockdown } & \multicolumn{2}{|c|}{ Post-lockdown } \\
\hline & Severity & $\begin{array}{l}\% \text { Experiencing } \\
\text { hassle }\end{array}$ & Severity & $\begin{array}{c}\% \text { Experiencing } \\
\text { hassle }\end{array}$ \\
\hline Inner concerns & $10.6(9.6)$ & 35.5 & $9.6(8.9)$ & 31.0 \\
\hline Time pressures & $8.2(7.2)$ & 33.7 & $9.1(7.5)$ & 38.1 \\
\hline Family hassles & $6.0(4.5)$ & 27.8 & $5.0(4.2)$ & 24.8 \\
\hline Financial concerns & $5.2(7.0)$ & 18.4 & $4.4(6.5)$ & 16.7 \\
\hline Health hassles & $3.0(3.4)$ & 19.9 & $2.8(3.4)$ & 19.2 \\
\hline Environmental hassles & $2.7(2.9)$ & 18.3 & $3.1(3.2)$ & 20.4 \\
\hline Work hassles & $2.4(3.4)$ & 17.6 & $2.7(3.4)$ & 20.3 \\
\hline
\end{tabular}

Higher numbers indicate higher severity of daily hassle category. 
TABLE 4 | Multiple regression results investigating relationships between daily hassles and PA behavior during-lockdown.

\begin{tabular}{|c|c|c|c|c|c|c|c|c|c|c|c|c|}
\hline & \multicolumn{4}{|c|}{ Model 1} & \multicolumn{4}{|c|}{ Model 2} & \multicolumn{4}{|c|}{ Model 3} \\
\hline & $\begin{array}{c}\text { Unstandardized } \\
\beta\end{array}$ & $\begin{array}{c}\text { Standardized } \\
\beta\end{array}$ & Sig. & VIF & $\begin{array}{c}\text { Unstandardized } \\
\beta\end{array}$ & $\begin{array}{c}\text { Standardized } \\
\beta\end{array}$ & Sig. & VIF & $\begin{array}{c}\text { Unstandardized } \\
\beta\end{array}$ & $\begin{array}{c}\text { Standardized } \\
\beta\end{array}$ & Sig. & VIF \\
\hline (Constant) & 994.34 & & 0.008 & & $1,081.09$ & & 0.005 & & $1,081.20$ & & 0.006 & \\
\hline $\begin{array}{l}\text { Pre-lockdown } \\
\text { total weekly PA }\end{array}$ & 0.41 & 0.44 & 0.00 & 1.09 & 0.40 & 0.44 & 0.00 & 1.10 & 0.40 & 0.44 & 0.00 & 1.99 \\
\hline Age & -3.14 & -0.06 & 0.34 & 1.03 & -3.25 & -0.06 & 0.32 & 1.03 & -3.25 & -0.06 & 0.33 & 1.04 \\
\hline Gender & 84.56 & 0.05 & 0.40 & 1.05 & 81.54 & 0.05 & 0.42 & 1.05 & 81.55 & 0.05 & 0.42 & 1.05 \\
\hline Education & 7.45 & 0.02 & 0.74 & 1.03 & 6.74 & 0.02 & 0.77 & 1.03 & 6.74 & 0.02 & 0.77 & 1.03 \\
\hline Ethnicity & -23.52 & -0.08 & 0.21 & 1.03 & -23.02 & -0.07 & 0.22 & 1.03 & -23.02 & -0.07 & 0.23 & 1.05 \\
\hline Essential worker & -46.29 & -0.02 & 0.72 & 1.02 & -47.61 & -0.02 & 0.71 & 1.03 & -47.63 & -0.02 & 0.71 & 1.03 \\
\hline Children & -32.68 & -0.04 & 0.47 & 1.04 & -33.15 & -0.05 & 0.46 & 1.04 & -33.15 & -0.05 & 0.46 & 1.04 \\
\hline $\begin{array}{l}\text { Extent of daily } \\
\text { hassles during } \\
\text { lockdown }\end{array}$ & & & & & -1.52 & -0.06 & 0.30 & 1.01 & -1.52 & -0.06 & 0.81 & 19.07 \\
\hline $\begin{array}{l}\text { Extent of daily } \\
\text { hassles during } \\
\text { lockdownPA } \\
\text { group }\end{array}$ & & & & & & & & & 0.004 & 0.05 & 1.00 & 19.25 \\
\hline
\end{tabular}

Dependent variable: total weekly PA during lockdown.

TABLE 5 | Multiple regression results investigating relationships between daily hassles and PA behavior post-lockdown.

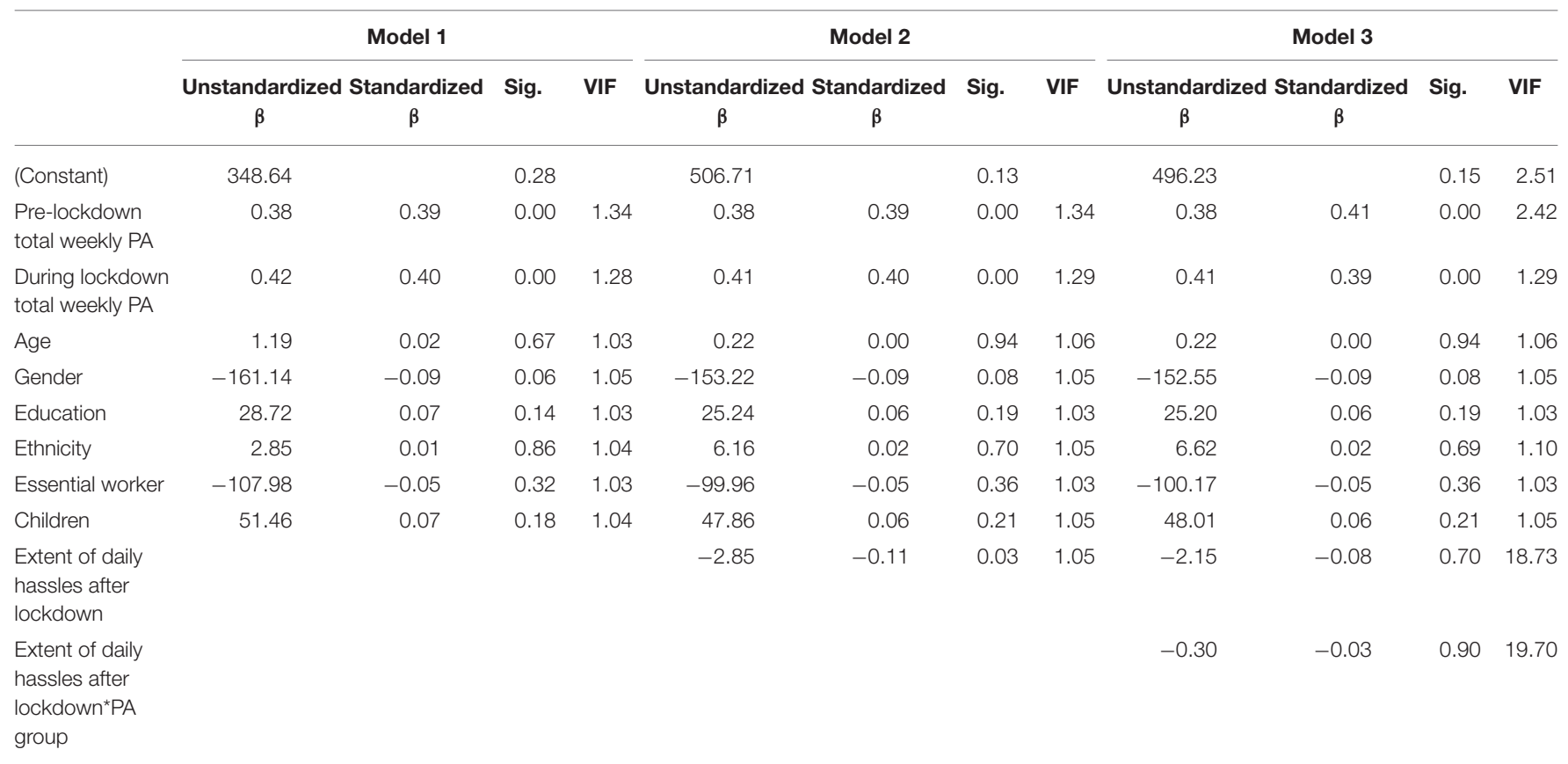

Dependent variable: total weekly PA after lockdown.

individuals who were highly active pre-lockdown, vigorous and moderate intensity PA was lower during- and post-lockdown compared to pre-lockdown, while walking behavior did not change. This meant that overall, compared to pre-lockdown, total PA was lower during- and post-lockdown in highly active individuals. Although, it is important to note that, despite PA reducing as a result of lockdown, on average these individuals were still exceeding recommended PA guidelines (World Health Organization, 2010). In comparison, for individuals who were moderately active pre-lockdown, overall PA, vigorous and moderate intensity PA was higher during-lockdown compared to pre-lockdown. Post-lockdown those increases in vigorous 
intensity PA remained while moderate intensity returned to prelockdown levels. Walking behavior was higher during-compared to post-lockdown. The results also showed that the severity of daily hassles was similar during- and post-lockdown. While daily hassles had a small, but significant association with PA behavior after lockdown, there was no association during lockdown.

Our PA results show the importance of analyzing PA data collected during COVID-19 restrictions according to how active people were prior to lockdown because the pattern of change differed between the highly active and moderately active groups. Previous COVID-related research reported decreased vigorous intensity PA (Cheval et al., 2020) and moderate-vigorous intensity PA (Di Sebastiano et al., 2020) during lockdowns, but did not account for changes across groups with different levels of pre-lockdown PA. Research which did account for pre-lockdown activity level found decreases in total PA (Barkley et al., 2020) and vigorous PA (Cheval et al., 2020) in highly active participants during-lockdown. Our results support these findings but also showed lockdown resulted in lower levels of moderate intensity PA than they would normally engage in (i.e., pre-lockdown). Reductions in PA may have been due to highly active individuals being unable to perform their preferred PA as a result of the lockdown restrictions. For example, gyms were closed, sport was canceled and people were confined to exercising close to home, which restricted people from long distance cycling or running. Not being able to do their preferred PA was reported by $45 \%$ respondents in our study when they were asked to explain why PA behavior had decreased (data available on request) and has been reported in other research with highly active individuals (e.g., Constandt et al., 2020; Kaur et al., 2020). Additionally, interviews with gym-goers in India, found the closure of gyms and parks resulted in a lack of "fitness motivation" and a need to find alternatives to their usual exercise routines (Kaur et al., 2020). The fact that PA levels of highly active individuals did not revert back to "normal" levels post-lockdown (even though exercise facilities were open and sport had resumed) could be attributed to the formation of new exercise habits during lockdown (Gardner and Rebar, 2019). Our second survey took place 6 weeks after lockdown ended and importantly, took place during the winter months (the pre-lockdown survey was administered during autumn). It may be that it takes longer than 6 weeks for individuals to readjust their behavior back to normal prelockdown PA levels or indeed, these highly active individuals have lower PA levels (different PA habits) during the winter months compared to autumn when the pre-lockdown behavior was assessed. Assessing PA again in autumn 2021 with no lockdown restrictions in place will provide data through which to evaluate the return to pre-lockdown PA behavior controlling for seasonal variations. An alternative explanation may be that, COVIDrelated changes in life situations (e.g., resumption of daily commute to work; changes in workplace or employment status; caregiving for children or family members) from pre- to postlockdown presented challenges to accumulating the previous large amounts of PA. In support of this explanation, having less time available for PA was reported by $57 \%$ of our respondents as an explanation for why their PA changed from during- to postlockdown. Inevitably, there will be a multitude of interconnected factors that explain PA changes during and after COVID-19 restrictions. Further research, employing qualitative methods, is needed to explore the key factors and interrelationships underpinning the changes.

Participants who were moderately active pre-lockdown increased their vigorous and moderate intensity PA levels duringlockdown, and importantly, maintained vigorous PA postlockdown. Consequently, total PA was higher post-lockdown compared to pre-lockdown. For moderately active participants, it appears lockdown provided an opportunity to increase the intensity of their pre-lockdown levels of PA. Explanations for these changes are speculative but it is possible that individuals wanted to use the time to "escape" from their homes and individuals knew being physically active was a permitted activity during-lockdown. Indeed, $35 \%$ of our participants who increased or maintained their PA levels during lockdown noted that being active "was a good excuse or reason to get outside". Participants may also have wanted to increase their fitness, and so chose to increase at a higher intensity to achieve that. Alternatively, moderately active individuals, compared to highly active individuals, may not have needed access to specialized facilities to significantly increase their PA levels. Being active close to home during-lockdown via simple modes of PA such as running and cycling, or at home using exercise equipment and online exercise videos [which was common during lockdown (Ding et al., 2020)], may have been attractive for these moderately active individuals. Although speculative, postlockdown, participants may have strengthened their autonomous motivation for PA (participating out of enjoyment, or the personal value attached), as a result of seeing the benefits and value of increasing their PA levels. Autonomous motivation is a strong predictor of PA behavior (Teixeira et al., 2012). Some evidence for this explanation was provided by participants who, in answer to the question "why have you been more active/continued to be active from during- to post-lockdown," reported that it was important for physical and psychological well-being. Other researchers have pointed to the importance of supporting motivation to enable PA behavior change during the pandemic (Hudson and Sprow, 2020; Matias et al., 2020). Given that the majority of research published so far examining PA during COVID-19 restrictions has been quantitative and largely descriptive, the explanations for why PA has changed have not been thoroughly explored, but clearly would be insightful. In particular, future research to understand changes in the different types of motivation people held for PA during the periods of COVID-19 restrictions would be of value.

This study also examined whether daily hassles might help to explain changes in PA behavior. The daily hassles (i.e., stressors) a specific life event creates in a person's life has been shown to influence lifestyle behaviors (Kanner et al., 1981; O'Connor et al., 2009; Uijtdewilligen et al., 2014). We found that inner concerns, time pressures, family hassles and financial concerns were experienced most severely and were endorsed by the greatest proportion of participants both during- and postlockdown. Contrary to hypotheses, the severity of daily hassles was not associated with PA during-lockdown. However, daily hassles had a small negative (Standardized $\beta=-0.11$ ) predictive 
effect on PA post-lockdown, which was not moderated by the participants' pre-lockdown PA status. Previous research has shown daily hassles to explain a similar proportion of variance in unhealthy snacking (Conner et al., 1999; O'Connor et al., 2009), smoking, fruit and vegetable consumption (O'Connor et al., 2009) and physical activity (Nguyen-Michel et al., 2006; O'Connor et al., 2009). While, feelings of worry/stress have been stated as reasons for decreasing engagement in positive health behaviors during lockdown (Knell et al., 2020), and those who reported decreases in PA during lockdown were more likely to report higher levels of stress (Stanton et al., 2020). It is unclear why daily hassles only predicted post-lockdown PA, even though the severity of daily hassles did not change from during- to postlockdown. It may be that the specific daily hassles encountered post-lockdown, compared to during-lockdown (e.g., returning to work, job insecurity), presented more of a challenge to PA levels. Investigating the relationships between daily hassles and the actual stress that resulted from those hassles may explain these findings.

This study is not without its limitations. We were not able to recruit many low active participants to complete the survey. The during-lockdown survey only garnered 32 responses from participants not meeting the PA guidelines, but they either did not opt to be contacted post-lockdown or did not respond to the post-lockdown survey and so could not be included in the analysis. Therefore, we have no data on how inactive people responded to the COVID-19 restrictions. Additionally, we had a low response rate to our post-lockdown survey which severely reduced our sample size for the longitudinal analysis, this presents a significant limitation. These selection biases have resulted in a sample with a specific demographic profile (predominately of NZ European ethnicity, female and highly educated) thus limiting generalisability of our findings. Our PA data is self-report, and for pre-lockdown behavior is retrospective, which comes with clear limitations in terms of recall bias, but is consistent with the methods of other research and indeed, the only method available under these circumstances. Despite these limitations, a strength of this research is the use of the validated IPAQ-SF as the self-report measure of PA and the longitudinal nature of the data.

In conclusion, contrary to what was predicted at the outset of the COVID-19 restrictions (Papaioannou et al., 2020), PA did not decline during lockdown restrictions in participants who were meeting the WHO PA guidelines prior to lockdown, in fact moderate and vigorous intensity PA increased. For those who were exceeding the guidelines prior to lockdown, PA declined but participation during lockdown was still at a

\section{REFERENCES}

Allender, S., Hutchinson, L., and Foster, C. (2008). Life-change events and participation in physical activity: a systematic review. Health Promot. Int. 23, 160-172. doi: 10.1093/heapro/dan012

Baltar, F., and Brunet, I. (2012). Social research 2.0: virtual snowball sampling method using facebook. Internet Res. 22, 57-74. doi: $10.1108 / 10662241211199960$ high enough level to benefit health. Post-lockdown, new PA habits had been created (highly active individuals pre-lockdown were participating in less PA compared to pre-lockdown, while moderately active individuals were more active) which may have been driven by changes in life circumstances or recognition of the importance of being physically active under COVID-19 conditions. As expected, participants reported experiencing daily hassles relating to inner concerns, family, time and finances as a result of COVID-19 restrictions, however it was only postlockdown that these hassles were negatively associated with PA. From a practical perspective, these results suggest that the information disseminated by the NZ government on the importance of remaining active during lockdown was heeded by active New Zealanders. Health promoters should publicize these positive results and encourage people to continue being active. Drawing on our daily hassles results, as New Zealand remains in a post-lockdown period, PA promotion information needs to communicate targeted strategies to overcome the daily hassles identified to provide on-going support for PA behavior. This could include emphasizing that being physically active can help reduce the experience of stress caused by daily hassles (World Health Organization, 2010; Stubbs et al., 2017). Future research should explore the potential explanations for why PA levels of active individuals were protected from the COVID19 restrictions. Furthermore, different methodologies that specifically target low active individuals are needed to explore potential changes in, and explanations for, their PA behavior.

\section{DATA AVAILABILITY STATEMENT}

The raw data supporting the conclusions of this article will be made available by the authors, without undue reservation.

\section{ETHICS STATEMENT}

The studies involving human participants were reviewed and approved by University of Otago, Human Ethics Committee. The patients/participants provided their written informed consent to participate in this study.

\section{AUTHOR CONTRIBUTIONS}

EH, MJ, and JC coordinated data collection. CL and JC cleaned and analyzed the data. EH led the manuscript writing. All authors contributed to the final submission, conceived the study, and developed the methods. 
Chen, P., Mao, L., Nassis, G. P., Harmer, P., Ainsworth, B. E., and Li, F. (2020). Coronavirus disease (COVID-19): the need to maintain regular physical activity while taking precautions. J. Sport Health Sci. 9, 103-104. doi: 10.1016/j.jshs.2020.02.001

Cheval, B., Sivaramakrishnan, H., Maltagliati, S., Fessler, L., Forestier, C., Sarrazin, P., et al. (2020). Relationships between changes in self-reported physical activity, sedentary behaviour and health during the coronavirus (COVID-19) pandemic in France and Switzerland. J. Sports Sci. 26, 1-6. doi: 10.1080/02640414.2020.1841396

Conner, M., Fitter, M., and Fletcher, W. (1999). Stress and snacking: a diary study of daily hassles and between-meal snacking. Psychol. Health 14, 51-63. doi: 10.1080/08870449908407313

Constandt, B., Thibaut, E., De Bosscher, V., Scheerder, J., Ricour, M., and Willem, A. (2020). Exercising in Times of lockdown: an analysis of the impact of COVID-19 on Levels and patterns of exercise among adults in Belgium. Int. J. Environ. Res. Public Health 17:4144. doi: 10.3390/ijerph171 14144

Craig, C. L., Marshall, A. L., Sjostrom, M., Bauman, A. E., Booth, M. L., Ainsworth, B. E., et al. (2003). International physical activity questionnaire: 12-country reliability and validity. Med. Sci. Sports Exerc. 35, 1381-1395. doi: 10.1249/01.MSS.0000078924.61453.FB

DeLongis, A., Coyne, J. C., Dakof, G., Folkman, S., and Lazarus, R. S. (1982). Relationship of daily hassles, uplifts, and major life events to health status. Health Psychol. 1:119. doi: 10.1037/0278-6133.1.2.119

Di Sebastiano, K. M., Chulak-Bozzer, T., Vanderloo, L. M., and Faulkner, G. (2020). Don't walk so close to me: physical distancing and adult physical activity in Canada. Front. Psychol. 11:1895. doi: 10.3389/fpsyg.2020.01895

Ding, D., Del Pozo Cruz, B., Green, M. A., and Bauman, A. E. (2020). Is the COVID-19 lockdown nudging people to be more active: a big data analysis. Br. J. Sports Med. 54, 1183-1184. doi: 10.1136/bjsports-2020-102575

Engberg, E., Alen, M., Kukkonen-Harjula, K., Peltonen, J. E., Tikkanen, H. O., and Pekkarinen, H. (2012). Life events and change in leisure time physical activity. Sports Med. 42, 433-447. doi: 10.2165/11597610-000000000-00000

Feizi, A., Aliyari, R., and Roohafza, H. (2012). Association of perceived stress with stressful life events, lifestyle and sociodemographic factors: a large-scale community-based study using logistic quantile regression. Comput. Math. Methods Med. 2012:151865. doi: 10.1155/2012/151865

Gardner, B., and Rebar, A. L. (2019). Habit Formation and Behavior Change. Oxford Research Encyclopedia of Psychology. Oxford: Oxford University Press. doi: 10.1093/acrefore/9780190236557.013.129

Godin, G., and Shephard, R. (1985). A simple method to assess exercise behavior in the community. Can. J. Appl. Sport Sci. 10, 141-146.

Holm, J. E., and Holroyd, K. A. (1992). The daily hassles scale (revised): Does it measure stress or symptoms? Behav. Assess. 14, 465-482. doi: 10.1037/t57783-000

Hudson, G. M., and Sprow, K. (2020). Promoting physical activity during the COVID-19 pandemic: implications for obesity and chronic disease management. J. Phys. Activity Health 9, 1-3. doi: 10.1123/jpah.2020-0318

IPAQ group (2014). Guidelines for Data Processing and Analysis of the International Physical Activity Questionnaire (IPAQ) - Short and Long Forms 2005. Available online at: https://sites.google.com/site/theipaq/scoring-protocol (accessed May 1, 2020).

Kanner, A. D., Coyne, J. C., Schaefer, C., and Lazarus, R. S. (1981). Comparison of two modes of stress measurement: daily hassles and uplifts vs. major life events. J. Behav. Med. 4, 1-39. doi: 10.1007/BF00844845

Kaur, H., Singh, T., Arya, Y. K., and Mittal, S. (2020). Physical fitness and exercise during the COVID-19 pandemic: a qualitative enquiry. Front. Psychol. 11:590172. doi: 10.3389/fpsyg.2020.590172

King, D. B., O'Rourke, N., and DeLongis, A. (2014). Social media recruitment and online data collection: a beginner's guide and best practices for accessing lowprevalence and hard-to-reach populations. Canad. Psychol. Psychol. Canad. 55, 240-249. doi: 10.1037/a0038087

Knell, G., Robertson, M. C., Dooley, E. E., Burford, K., and Mendez, K. S. (2020). Health behavior changes during COVID-19 pandemic and subsequent "stay-at-home" orders. Int. J. Environ. Res. Public Health 17:6268. doi: 10.3390/ijerph17176268

Lee, P. H., Macfarlane, D. J., Lam, T. H., and Stewart, S. M. (2011). Validity of the international physical activity questionnaire short form
(IPAQ-SF): a systematic review. Int. J. Behav. Nutr. Phys. Activity 8:115. doi: 10.1186/1479-5868-8-115

Lesser, I. A., and Nienhuis, C. P. (2020). The impact of COVID-19 on physical activity behavior and well-being of Canadians. Int. J. Environ. Res. Public Health 17:3899. doi: 10.3390/ijerph17113899

Lippi, G., Henry, B. M., and Sanchis-Gomar, F. (2020). Physical inactivity and cardiovascular disease at the time of coronavirus disease 2019 (COVID-19). Eur. J. Prev. Cardiol. 27, 906-908. doi: 10.1177/2047487320916823

López-Bueno, R., Calatayud, J., Andersen, L. L., Balsalobre-Fernández, C., Casaña, J., Casajús, J. A., et al. (2020). Immediate impact of the COVID-19 confinement on physical activity levels in Spanish adults. Sustainability 12:14. doi: $10.3390 /$ su12145708

Matias, T., Dominski, F. H., and Marks, D. F. (2020). Human needs in COVID-19 isolation. J. Health Psychol. 25, 871-882. doi: 10.1177/1359105320925149

Matthews, C. E., Steven, C. M., George, S. M., Sampson, J., and Bowles, H. R. (2012). Improving self-reports of active and sedentary behaviors in large epidemiologic studies. Exerc. Sport Sci. Rev. 40:118. doi: 10.1097/JES.0b013e31825b34a0

Meyer, J., McDowell, C., Lansing, J., Brower, C., Smith, L., Tully, M., et al. (2020). Changes in physical activity and sedentary behavior in response to COVID-19 and their associations with mental health in 3052 US adults. Int. J. Environ. Res. Public Health 17:6469. doi: 10.3390/ijerph17186469

Mutz, M., and Gerke, M. (2020). Sport and exercise in times of self-quarantine: how Germans changed their behaviour at the beginning of the Covid-19 pandemic. Int. Rev. Sociol. Sport. 1-12. doi: 10.1177/1012690220934335

New Zealand Government (2020a). New Zealand COVID-19 Alert Levels. Wellington.

New Zealand Government, (2020b). Health Act (COVID-19 Alert Level 3) Order. Wellington.

Nguyen-Michel, S. T., Unger, J. B., Hamilton, J., and Spruijt-Metz, D. (2006). Associations between physical activity and perceived stress/hassles in college students. Stress Health 22, 179-188. doi: 10.1002/smi.1094

O'Connor, D. B., Conner, M., Jones, F., McMillan, B., and Ferguson, E. (2009). Exploring the benefits of conscientiousness: an investigation of the role of daily stressors and health behaviors. Ann. Behav. Med. 37, 184-196. doi: 10.1007/s12160-009-9087-6

O'Connor, D. B., Jones, F., Conner, M., McMillan, B., and Ferguson, E. (2008). Effects of daily hassles and eating style on eating behavior. Health Psychol. 27:S20. doi: 10.1037/0278-6133.27.1.S20

Papaioannou, A. G., Schinke, R. J., Chang, Y. K., Kim, Y. H., and Duda, J. L. (2020). Physical activity, health and well-being in an imposed social distanced world. Int. J. Sport Exerc. Psychol. 18, 414-419. doi: 10.1080/1612197X.2020.1773195

Ricci, F., Izzicupo, P., Moscucci, F., Sciomer, S., Maffei, S., Di Baldassarre, A., et al. (2020). Recommendations for physical inactivity and sedentary behavior during the coronavirus disease (COVID-19) pandemic. Front. Public Health 8:199. doi: 10.3389/fpubh.2020.00199

Salari, N., Hosseinian-Far, A., Jalali, R., Vaisi-Raygani, A., Rasoulpoor, S., Mohammadi, M., et al. (2020). Prevalence of stress, anxiety, depression among the general population during the COVID-19 pandemic: a systematic review and meta-analysis. Global. Health 16:57. doi: 10.1186/s12992-020-00589-w

Sallis, J. F., Adlakha, D., Oyeyemi, A., and Salvo, D. (2020). An international physical activity and public health research agenda to inform coronavirus disease-2019 policies and practices. J. Sport Health Sci. 9, 328-334. doi: $10.1016 /$ j.jshs.2020.05.005

Sallis, J. F., and Pratt, M. (2020). Why Keep This COVID-19 Remedy a Secret. Available online at: https://www.exerciseismedicine.org/support_page. $\mathrm{php} /$ stories/?b=912 (accessed October 27, 2020).

Schnitzer, M., Schottl, S. E., Kopp, M., and Barth, M. (2020). COVID-19 stay-athome order in Tyrol, Austria: sports and exercise behaviour in change? Public Health 185, 218-220. doi: 10.1016/j.puhe.2020.06.042

Simpson, R. J., and Katsanis, E. (2020). The immunological case for staying active during the COVID-19 pandemic. Brain Behav. Immun. 87, 6-7. doi: 10.1016/j.bbi.2020.04.041

Stanton, R., To, Q. G., Khalesi, S., Williams, S. L., Alley, S. J., Thwaite, T. L., et al. (2020). Depression, anxiety and stress during COVID-19: associations with changes in physical activity, sleep, tobacco and alcohol use in Australian adults. Int. J. Environ. Res. Public Health 17:4065. doi: 10.3390/ijerph1711 4065 
Stubbs, B., Vancampfort, D., Rosenbaum, S., Firth, J., Cosco, T., Veronese, N., et al. (2017). An examination of the anxiolytic effects of exercise for people with anxiety and stress-related disorders: a meta-analysis. Psychiatry Res. 249, 102-108. doi: 10.1016/j.psychres.2016.12.020

Stults-Kolehmainen, M. A., and Sinha, R. (2014). The effects of stress on physical activity and exercise. Sports Med. 44, 81-121. doi: 10.1007/s40279-013-0090-5

Teixeira, P. J., Carraça, E. V., Markland, D., Silva, M. N., and Ryan, R. M. (2012). Exercise, physical activity, and self-determination theory: a systematic review. Int. J. Behav. Nutr. Phys. Activity 9:78. doi: 10.1186/1479-5868-9-78

Twisk, J. W., Snel, J., Kemper, H. C., and van Mechelen, W. (1999). Changes in daily hassles and life events and the relationship with coronary heart disease risk factors: a 2-year longitudinal study in 27-29-year-old males and females. J. Psychosom. Res. 46, 229-240. doi: 10.1016/S0022-3999(98)00088-9

Uijtdewilligen, L., Singh, A. S., Chinapaw, M. J., Koppes, L. L., van Mechelen, W., and Twisk, J. W. (2014). Number and appraisal of daily hassles and life events in young adulthood: the association with physical activity and screen time: a longitudinal cohort study. BMC Public Health 14:1067. doi: 10.1186/1471-2458-14-1067

Wilson, N., Grout, L., Summers, J., Nghiem, N., and Baker, M. (2020). Health and Economic Impacts of the COVID-19 Response: NZ Compared to OECD Countries. Available online at: https://blogs.otago.ac.nz/pubhealthexpert/?p= 8818 (accessed December 4, 2020).
Woods, J. A., Hutchinson, N. T., Powers, S. K., Roberts, W. O., GomezCabrera, M. C., Radak, Z., et al. (2020). The COVID-19 pandemic and physical activity. Sports Med. Health Sci. 2, 55-64. doi: 10.1016/j.smhs.2020. 05.006

World Health Organisation (2020). WHO Announces COVID-19 Outbreak a Pandemic. Available online at: https://www.euro.who.int/en/healthtopics/health-emergencies/coronavirus-covid-19/news/news/2020/3/whoannounces-covid-19-outbreak-a-pandemic (accessed May 21, 2020).

World Health Organization (2010). Global Recommendations on Physical Activity for Health. Geneva: World Health Organization.

Conflict of Interest: The authors declare that the research was conducted in the absence of any commercial or financial relationships that could be construed as a potential conflict of interest.

Copyright $\odot 2021$ Hargreaves, Lee, Jenkins, Calverley, Hodge and Houge Mackenzie. This is an open-access article distributed under the terms of the Creative Commons Attribution License (CC BY). The use, distribution or reproduction in other forums is permitted, provided the original author(s) and the copyright owner(s) are credited and that the original publication in this journal is cited, in accordance with accepted academic practice. No use, distribution or reproduction is permitted which does not comply with these terms. 